\title{
Milliliter per Minute per Milligram per Meter Squared
}

National Cancer Institute

\section{Source}

National Cancer Institute. Milliliter per Minute per Milligram per Meter Squared. NCI

Thesaurus. Code C120795.

A unit of concentration equal to milliliter per minute divided by milligram per meter squared. 